Sakai and Toquenaga 1

\title{
Heterostyly: Speciation within a species
}

Shoko SAKAI and Yukihiko TOQUENAGA

University of Tsukuba, Institute of Biological Sciences, Tsukuba, Ibaraki, 305-8572, Japan

Author for correspondence

Shoko SAKAI

Current address: Center for Ecological Research, Kyoto University, Otsu 520-2113, Japan.

Tel. $+81-77-549-8260$

Fax. $+81-77-549-8201$

e-mail: shoko@biol.tsukuba.ac.jp 


\begin{abstract}
Almost all the organisms in nature show non-random mating in different degrees. Two extreme results of nonrandom mating are speciation and sexual differentiation. Heterostyly is a form of sexual differentiation considered to have evolved to resolve conflicts between male and female functions of hermaphrodite flowers. Our study examines necessary and sufficient conditions for establishment of heterostyly using a configuration individual-based model. Previous models assume invasion of a mutant phenotype into a population with monomorphic wild phenotype. In contrast, our model demonstrated that heterostyly could establish from a population with continuous phenotypic variation, which requires more simple assumptions than the previous hypotheses. Results of our simulation show that genetic linkage between stigma and anther heights is essential for establishment of heterostyly. Dominance effects on the genes for stamen or stigma heights are not necessary, but they promote evolution of heterostyly. Probability of evolution of heterostyly also depends on functional relationship between stigma-anther distance and strength of sexual interference, and the distance and probability of pollen deposition success. Parallelity and difference between speciation and sexual differentiation are also discussed.
\end{abstract}

Key word breeding system, individual-based model, nonrandom mating, sexual dimorphism, sexual interference 


\section{Introduction}

Almost all the organisms in nature show nonrandom mating in different degrees. Two extreme results of nonrandom mating are speciation and sexual differentiation. Preference for mates with characteristics similar to those of themselves (assortative mating) promotes differentiation within species, which may end up with breakups into two or more reproductive groups or "species" (e.g. Higashi et al. 1999). Tendency to mate between dissimilar individuals, on the other hand, may lead to sexual differentiation. However, continuity from speciation to sexual differentiation, underlining theme of this paper, has rarely been discussed.

Independent from our study, Bolnick and Doebeli (2003) give an attention on the continuity, and argue that sexual dimorphism and adaptive speciation is two sides of the same ecological coin. The study shows that disruptive selection due to frequency-dependent interactions can lead to either speciation or sexual dimorphism depending on the genetic independence of male and female traits and the potential strength of assortative mating. Our model is qualitatively different from Bolnick and Doebeli (2003), in which male and female already exist at the start, in that populations with no sexual differentiation diverge into two sexual morph types under certain conditions. The difference is partly because they presume sexual dimorphism of animals with rather clear "male" and female" distinction, but we do that of flowering plants, most of which are hermaphrodite (Richards 1997).

In contrast to dioecious plants and animals, outcrossing hermaphrodite plants suffer significant conflicts between male and female function of their flowers to disperse and to receive pollen (Barrett 2002b). Although the principal cost of 
hermaphrotism is self-fertilization and the reduced fitness of offspring resulting from inbreeding depression, male-female interference within an individual or "sexual interference" can occur through different mechanisms (Barrett 2002b). Increasing evidences suggest the presence of self pollen on stigmas physically and/or physiologically interfere cross-pollen-tube growth and cross fertilization (pollen clogging) in self-incompatible plants (e.g. Bertin and Sullivan 1988; Waser and Price 1991; Broyles and Wyatt 1993). Late acting self-incompatibility with self rejection in the ovary after fertilization, or inbreeding depression in self-compatible plants also causes wastage of ovules and seeds (ovule and seed discounting) (Waser and Price 1991; Brpoyles and Wyatt 1993; Seavey and Carter 1993). Deposition of pollen on stigmas of the same flower or within the individual bring about reduction of pollen available for outcrossing (pollen discounting; "pollen saving" in Charlesworth and Charlesworth 1978) (Hader and Barrett 1995; Harder and Wilson 1998). To compromise the two inconsistent functions or male and female, hermaphrodite flowers have evolved different mechanisms including the separations of stigma receptivity and pollen release within a flower in space (herkogamy) and time (dichogamy), which are very common characters among hermaphroditic plants. Although such mechanisms are often interpreted to be against selfing, it has been recognized that reduction of sexual interference is more complete explanation (Barrett 2002b).

Heterostyly is a form of sex differentiation considered to have evolved to resolve conflicts between male and female functions of hermaphrodite flowers (Barrett 2002a). In heterostylous plants, populations are composed of two (in the case of distyly) or three morphs (tristyly) that differ reciprocally in the height at which stigmas and anthers are positioned in flowers (Fig. 1). The floral morph with stigmas exerted above 
the stamens is called "pin," and the other morph with lower stigmas and higher anthers is "thrum." Distyly is simply inherited with a single diallelic Mendelian locus with dominance, while two diallelic locuses both with dominance with epistatical interaction between them govern tristyly. The stigma-anther height polymorphisms is usually accompanied by a sporophytically controlled, diallelic self-incompatibility system that prevents self and intramorph fertilizations (Barrett 1992; Barrett 2002a). Since general properties of their diallelic sporophytic incompatibility system are fundamentally distinct from those of gametophytic multiallelic incompatibility found in most self-incompatible plants, the former is believed to have a different origin from the latter (Barrett 2002a). It supports the view that heterostyly has arisen from self-compatible plants.

Heterostyly has evolved in at least 28 animal-pollinated angiosperm families independently (Barrett 2002a), but heterostylous plants are not distributed at random at all (Lloyd and Webb 1992a) and are still minority in the whole Angiosperms. Ganders (1979) lists 155 genera including heterostylous species, which are 1-2\% of the genera of flowering plants, and more than a half of the heterostylous genera are in Rubiaceae (Bawa and Beach 1983). Necessary and sufficient conditions for establishment of heterostyly, however, have not been examined sufficiently, and theoretical studies so far postulate an evolutional scenario of heterostyly a priori, and appeal explicability of the scenario analytically (see Discussion). It is partly due to limitation of analytical approach to deal with a system in which reproductive success of an individual is strongly dependent on not only phenotype of itself but also phenotypes of other individuals within the population. In a similar case of speciation, individual-based model has been proved to be a strong tool (e.g. Dieckmann and Doebeli 1999; Higashi 
et al. 1999; Bolnick and Doebeli 2003), although it has rarely been used to examine evolution of plant breeding systems. With the model we can more simply and directly express dependency of reproductive success of an individual on the phenotypes of potential mates through pollination success and through phenotype of offspring than with analytical approaches. Besides, the model enables us to explicitly define expression and inheritance of genes, and evaluate the effects of genetic systems qualitatively and quantitatively. Those evaluations are indispensable to link molecular genetics of phenotypic traits and evolution of sexual systems.

In this study, we examine conditions for establishment of heterostyly using a configuration individual-based model (Kawata and Toquenaga 1994). Our model assumes that reproductive success of an individuals depends on stigmas and anthers heights of itself and those of potential mates: stigma and anthers close to each other within a flower cause decrease in reproductive success due to sexual interference, and flowers are successfully pollinated when anther height of the pollen donor is close to stigma height of the recipient. We use two function sets arbitrary chosen to express the dependency (see Model). For simplicity, we do not consider mutation or crossing over in this model. Instead, the initial populations are constituted of individuals with stigma and anther heights of unimordal distribution, and their combinations are random. Our analyses demonstrate presence of sexual-interference and higher pollination success between anther and stigma of similar heights do not always bring about evolution of heterostyly. It depends on functional relationships between stigma and anther heights and reproductive success. In addition, we conducted simulations under three different assumptions of genetic systems of the phenotypes: (1) assumption without genetic linkage between stigma and anther height or dominance effects, (2) with linkage effect 
but without dominance effects, and (3) with linkage and dominance effect on genes for stigma and anther heights. The results show that genetic linkage between stigma and anther heights is essential for establishment of heterostyly, but dominance effects on the genes for stamen or stigma heights are not necessary.

\section{Model}

We conducted simulations using a configuration individual-based model to investigate conditions for establishment of heterostyly from a population with anther and stigma heights of unimordal distribution (see below). Our models assume discrete generations, and random visitation of pollinators to each plant. Neither variation in plant size nor special structure is considered. The initial populations have 300 individuals, and the numbers are maintained to be equal to or below the initial ones by randomly removing excess individuals. We conducted 200 replicate simulations each under six different conditions (Table 1 and see below). All programs were written in Ruby script (Matsumoto and Reynolds 2001; Matsumoto 2002) running on a MacOS X 10.2 machine.

Sexual interference and pollen deposition success: two conditions

Reproductive success through pollen and seeds mediated by pollinators depend on two phenotypic values, anther and stigma heights ( $H_{s}$ and $H_{a}$, both range from 0.0 to 1.0), of themselves and potential mates. Each individual has potential to produce $S$ seeds, while the number can be lower due to sexual interference on female function, which 
depends on stigma-anther separation within a flower $\left(x_{\text {self }}=\left|H_{s}-H_{a}\right|\right)$, or pollen limitation (see below). We assume monotonous increase of seed production (when pollen does not limit seed set) with $x_{\text {self }}$ (Fig. 2).

$P$ pollen donors are randomly chosen for each maternal plant (pollen recipient), while success of pollination depends on stigma-anther separation ( $\left.x_{\text {self }}\right)$ of the pollen donor and the difference of anther height of the pollen donor and stigma height of the recipient $\left(x_{\text {mate }}\right)$. First, we again assume monotonous increase of probability of successful pollen removal with $x_{\text {self }}$ of pollen donor (Fig. 2), because small stigma-anther separation $\left(x_{\text {self }}\right)$ of the pollen donor interferes pollen removal by pollinators. Second, pollen deposition on the recipient stigma tends to succeed when the difference of anther height of pollen donor and stigma height of the recipient $\left(x_{\text {mate }}=\left|H_{\text {s(recipient })}-H_{a(\text { donor })}\right|\right)$ is small enough, because pollen is deposited only on particular part of the pollinator body (Fig. 1). Thus, probability of pollen deposition success decreases with $x_{\text {mate }}$ (Fig. 2). A single successful pollen transfer deposits a single pollen grain on the stigma, and pollen grains on the stigma fertilize ovules to produce seeds in a random order until the seed number reaches its maximum determined by the potential seed production $S$ and $x_{\text {self }}$ of the recipient as described above.

For relationships between $x_{\text {self }}$ and sexual interference on female (decrease of seeds) and male (less pollen removal) functions, and between $x_{\text {mate }}$ and probability of successful pollen deposition, we adopt two sets of functions. Although it is not necessary we use the same function to express (1) increase of pollen removal probability with $x_{\text {self }},(2)$ increase of seed set with larger $x_{\text {self }}$ (Figs. 2a(1) and 2b(1)) and increase of failure of pollen deposition (= 1- successful pollen deposition) with $x_{\text {mate }}$ (Figs. 2a(2) and $2 \mathrm{~b}(2)$ ). In the linear assumption (Fig. 2a), an individual with $x_{\text {self }}$ 
produces $S \cdot x_{\text {self }}$ seeds when pollination does not limit seed set, and probability of successful pollen removal is $x_{\text {self }}$. Probability of successful pollen deposition is $1-x_{\text {mate }}$. Under the square-root assumption (Fig. 2b), which assumes stronger effects of distances when the distances are smaller, an individual with $x_{\text {self }}$ produces $S \cdot \sqrt{x_{\text {self }}}$ seeds when pollination does not limit seed set, and probability of pollen removal is $\sqrt{x_{\text {self }}}$. Probability of successful pollen deposition is $1-\sqrt{x_{\text {mate }}}$. Parameters $S$ and $P$ were chosen so that population size rarely drops below the initial one and that durations of simulations are as short as possible: $S=10$ and $P=10$ for the linear assumption, and $S=6$ and $P=6$ for the square-root assumption.

Genetic system: three conditions

We employed a diploid genetic system. In the most simple genetic system (Condition 1 and 2 in Table 1), each of the two phenotypic values, stigma and anther heights, is an average of two genetic values ( $s_{1}$ and $s_{2}$ for stigma height and $a_{1}$ and $a_{2}$ for anther height) ranging 0.0-1.0 (Fig. 3a). Random real numbers are assigned to the four genetic values of individuals in the initial populations, therefore stigma and anther heights of the initial population become unimodal distributions (cf. the central limit theorem).. One of the two genetic values for stigma and anther height of a parent is randomly transmitted to offspring. Each of those real values emulate additive effects of multiple genes on a single chromosome.

In the second genetic system (Condition 3 and 4 in Table 1), we incorporated associated transmission of anther and stigma genes (linkage). We propose an additive genetic system for linkage as well as dominance (see below). Probability of associated 
inheritance of genetic values for stigma and anther heights is defined as an average of two additional genetic values for linkage strength $\left(l_{1}\right.$ and $\left.l_{2}\right)$ of the individual. When $l_{1}=l_{2}=1$, genetic values for stigma $\left(s_{1}\right)$ and anther heights $\left(a_{1}\right)$ from its father (or $s_{2}$ and $a_{2}$ from mother) are always transmitted together to its offspring. When $l_{1}=l_{2}=0$, genetic values for stigma and anther heights from parents are transmitted randomly. When the value is between 0 and 1, the genetic values show associated inheritance with the probability depending on the linkage strength values. If the linkage strength $\left(\frac{l_{1}+l_{2}}{2}\right)$ is 0.2 , the values are transmitted together in $20 \%$ cases, and in $80 \%$ the value is transmitted randomly ( $s_{1}$ may be transmitted together with $a_{1}$ or $a_{2}$ with the same probability). Random real numbers from 0 to 1 are assigned to the two linkage genetic values of individuals in the initial populations, and the values themselves are randomly inherited from parents to offspring.

In the third system (Conditions 5 and 6 in Table 1), we incorporated dominance effects in addition to the linkage. When genetic values for stigma heights are $s_{1}$ and $s_{2}\left(s_{1}<s_{2}\right)$, and genetic values for dominance effect on stigma are $d_{s 1}$ and $d_{s 2}$, stigma height is defined as $s_{1}+\left(s_{2}-s_{1}\right) \frac{\left(d_{s 1}+d_{s 2}\right)}{2}$ (Fig. 3b). Dominance effect on anther height is also defined in the same way by the other two genetic values ( $d_{a 1}$ and $d_{a 2}$ ). Random real numbers from 0 to 1 are assigned to the four dominance genetic values of individuals in the initial populations, and the values are randomly inherited from parents to offspring.

Classification of resulting populations 
During simulations we examined the status of the populations every 20 generations, and quitted the run when variation of genetic values for stigma and anther heights became too low to establish heterostyly ("low variation"), or when heterostyly was established ("heterostyly"). Otherwise, the run was continued until it reached the 5000th generation. When either of the two following conditions was satisfied, the population was classified into "low variation": range of genetic value for stigma or stamen height is less than 0.025 ; or the maximum genetic value for anther (stigma) height is smaller than minimum genetic value for stigma (anther) height. To judge establishment of heterostyly, we examined distribution of combinations of stigma and anther heights on two-dimensional $20 \times 20$ histogram. When the two cells including the most and the second most individuals represent two morphs with stigmas and anthers reciprocally arranged, in other word the locations of the two cells, $\left(H_{s 1}, H_{a 1}\right)$ and $\left(H_{s 2}, H_{a 2}\right)$, meet the conditions $\left(H_{s 1}-H_{s 2}\right) \cdot\left(H_{a 1}-H_{a 2}\right)<0$ and $\operatorname{Cov}\left(H_{s}, H_{a}\right)<0$ in five consecutive examinations (Fig. 4), the population was judged to be heterostylous. When heterosyly establishes, differences in stigma and anther heights between the two morphs were calculated as $\left|H_{s 1}-H_{s 2}\right|$ and $\left|H_{a 1}-H_{a 2}\right|$, respectively.

\section{Results}

Without the linkage between the genetic values for stigma and anther heights, heterostyly never evolved (Table 1). Under the linear assumption (Condition 1), variation in the genetic values was quickly lost and runs were stopped at the 160th generation or earlier. Phenotypic values of the individuals of the final populations were concentrated on the line $\mid H_{s}-H_{a} \models 0.5$ (Fig. 5a). Under the square-root assumption 
(Condition 2), loss of genetic variation occurred slower, and runs were continued to the 5000th generations with significant genetic variation maintained in the replications of $18 \%$. Phenotypic values of individuals of the final populations were concentrated on the line $\mid H_{s}-H_{a} \models 0.25$ (Fig. 5b).

When the linkage effect was incorporated, variation was quickly lost again within 160 generations when the functions were assumed to be linear (Condition 3). However, heterostyly was established in $62 \%$ of populations under the square-root assumption (Condition 4). In those heterostylous populations, linkage was always selected for (Table 1, Fig. 6). Individuals of one morph were heterozygous in the genetic values for both anther and stigma heights, and those of the other were homozygous (Fig. 6). Differences of stigma and anther heights between the two morphs in the heterostylous populations were $0.40 \pm 0.06$ and (Mean \pm SD and hereafter) $0.29 \pm$ 0.07 , respectively.

Dominance effects greatly improved the probability of establishment of heterosyly. Even under the linear assumption (Condition 5), $65 \%$ of the populations evolved to be heterostylous. Under the square-root assumption, the proportion reached as high as $87 \%$, and time to reach heterostyly was shorter than simulations without dominance effects (Table 1, $P=0.0017$, t-test, two-sided, hereafter). Differences of stigma and anther heights between the two morphs in the heterostylous populations were much larger under the linear assumption (stigma, $0.85 \pm 0.06$; anther, $0.82 \pm 0.09$ ) than under the square-root assumption (stigma, $0.64 \pm 0.09$; anther, $0.59 \pm 0.11$ ) (for both stigma and anther heights, $P<0.0001)$. In heterostylous populations, dominance effects on anther and stigma heights were selected to opposite direction (Fig. 7). 


\section{Discussion}

Two major gaps in our knowledge seriously restrict understanding of the evolution of heterostyly (Barrett 2000). First, we know nothing about molecular and developmental genetics of the polymorphism. Despite of rapid increase of studies on genetic controls of phenotypic traits, we know little about evolution of quantitative traits of flowers. A few available studies using quantitative trait locus (QTL) mapping approach indicate that variation of quantitative floral characters is controlled by different genes with different magnitudes of effects. Genetic correlations between traits are usually positive and high, suggesting either pleiotropy or tight linkage (Juenger et al. 2000; Hodges et al. 2002). Phenotypic differences in closely related species can be explained by a few QTLs or have highly polygenic basis (e.g. Bradshaw 1995; Fishman et al. 2002; reviewed in Orr 2001). In addition, it is very difficult to clearly determine the ancestral condition even with cladistic approach without examining genes responsible for differences among related species and their changes through evolution and speciation. In such a case, it is a fruitful approach to construct a model with as few assumptions as possible.

We examine if the two genetic effects characterizing genetic system of heterostyly, strong linkages among genes related with the heteromorphy (often called super gene) and dominance of one of the two alleles of the super gene, are necessary for the establishment of heterostyly. Results of the simulations show that linkage between genes for stigma and anther heights are necessary conditions for evolution of heterosyly, but dominance effects are not. In the case of the speciation, genetic association between preference and a maker trait to select a mate may be formed and strengthened through 
assortative mating. Disruptive selection can be caused by the preference and maker themselves (Higashi et al. 1999), or other ecological traits (Kondrashov and Kondrashov 1999; Dieckmann and Doebeli 1999). In heterostyly, on the other hand, the cause of disruptive selection is sexual interference (Table 2), and the individuals prefer to mate with opposite phenotype. Due to this disassotative mating, a mating pair has opposite preference and maker traits. Therefore, some mechanisms, such as linkage, is required to distribute appropriate combinations of their preference and maker genes to offspring.

With the linkage effect, heterosyly established when the function between stigma-anther separation and sexual interference, and the function between difference between anther height of pollen donor and stigma height of recipient and pollen deposition success were square root (Condition 4), but not when the two functions were linear (Condition 3). The most important difference between the two functional models may be optimal stigma-anther distances. When there is no linkage, a population tends to converge to a morph with an optimal difference between stigma and anther heights. The optimal difference is 0.5 under the linear assumption, since the product of effects of sexual interference and pollen deposition success, both of which are functions of stigma-anther height difference, maximizes when the difference is 0.5 (Fig. 2a). Thus individuals at the final generation under the linear assumption are concentrated on the line of $\mid H_{s}-H_{a} \models 0.5$ (Fig. 5a). On the other hand, the optimal distance is 0.25 under the square-root assumption (Figs. 2b), and individuals at the final generation under the square-root assumption are concentrated on the line of $\mid H_{s}-H_{a} \models 0.25$ (Fig. 5b).

Genetic system of heterostyly without dominance effects, sets some limits on stigma-anther distance. Let us consider a heterostylous population with genetic values 
for stigma height $s l$ and $s_{s}$ and values for anther $a_{l}$ and $a_{s}$, which have size relationships as follows.

$$
\begin{aligned}
& s_{l}>s_{s} \\
& a_{l}>a_{s}
\end{aligned}
$$

Assuming that pin morph is heterozygous and thrum homozygous, and that stigma and anther height of pin are $\frac{s_{l}+s_{s}}{2}$ and $\frac{a_{l}+a_{s}}{2}$, those of thrum should be $s_{s}$ and $a_{l}$, since stigma of pin morph is higher than that of thrum, and it should be reverse in anther height. From the assumption of heterostyly that stigma height of pin morph is equal to anther height of the thrum morph and vise versa, the values have the following relationships.

$$
\begin{aligned}
& \frac{s l+s s}{2}=a_{l} \\
& \frac{a_{l}+a_{s}}{2}=s_{s}
\end{aligned}
$$

From the inequalities and equations (1)-(4), the genetic values should satisfy an inequality $s_{l}>a_{l}>s_{s}>a_{s}$. Let $s_{l}=1$ and $a_{s}=0$ to maximize stigma-anther distance. Then we get stigma and anther height of pin flowers $\frac{2}{3}$ and $\frac{1}{3}$, and those for thrum flowers, which are opposite of the pin. The same is true if we assume thrum to be heterozygote instead of pin. Therefore, maximum stigma-anther distance is $\frac{1}{3}$. Because the optimal distance under the linear assumption, 0.5 , is out of the range that the genetic system allows, dominance of a single morph with the optimal stigma-anther distance does not followed by counterbalancing increase of other morph with anther and stigma of reciprocal arrangement, and heterostyly does not evolve. Contrarily, under the square root assumption, dominance of a single homozygous morph promotes to build up a 
complementary heterozygous morph, which shares one of the two alleles with homozygous morph, resulting in heterostyly. The difference between the two assumptions is caused by different optimal distances rather than that of the shape (linear or square-root) of the functions themselves.

Introduction of dominance effects enables establishment of heterostyly under the linear assumption (Condition 5). In this case, stigma and anther heights of extreme values (anther and stigma heights close to 0 or 1 ) are selected for, because the initial populations have anther and stigma heights with a peak at 0.5 . The shift to a bimodal distribution is quickly followed by selection on dominance effects and linkage strength, and by establishment of heterostyly (Fig. 6). On the other hand, the first selection under the square-root assumption (Condition 6) drives stigma and anther heights to be around 0.25 and 0.75 , since optimal stigma-anther distance is 0.25 . Therefore resulted heterostylous populations under the square-root assumption have shorter stigma-anther distance than those under the linear assumption.

Our model is fundamentally different from previous ones in that we do not assume invasion of a mutant with drastically different phenotype from normal ones (Fig. 8). Ganders (1979) and Lloyd and Webb (1992a) presume that ancestors of heterostylous species had flowers of approach herkogamy (flowers with stigmas above and separated from anthers, morphologically similar to pin in heterostylous plant but monomorphic) (Fig. 8a), partly because plants with stigmas and anthers with little spatial separation is usually found in self-pollinated plants, in which selection to promote outcrossing is unlikely to be strong enough for establish heterostyly. In addition, compared to reverse herkogamy (flowers with anthers above and separated from stigmas), approach herkogamy is widespread among outcrossing angiosperms. 
Lloyd and Webb (1992a, b) colloquially propose a sequence of the establishment of heterostyly from approach herkogamy, style polymorphis, to reciprocal herkogamy (heterostyly) (Fig. 8a), and mathematically show that a mutant can spread into a uniform normal type population when proficiency of pollen transfer between a normal type and the mutant is higher than that between two normal types. Interestingly, they argue that heteromorphic incompatibility is due to intramorph failure arising incidentally from specialization for intermorph pollinations as Darwin postulated, in addition to active selection restricting self-fertilization. The process may be almost identical to putative establishment of an interspecific barrier in speciation.

It is quite possible to examine previous models by incorporating additional parameters or changing initial population characters. This study provides a platform to develop inclusive models rather than opposing to the previous ones. For example, effects of different distributions of stigma and anther heights in initial populations on establishment of heterostyly is clearly one of the important subjects of future studies. In this paper, we randomly assigned genetic values for initial individuals. Therefore, averages of both stigma and anther heights are equal to 0.5 , and it is against the argument of Lloyd and Webb (1992a). However, we do not think it unreasonable to postulate that ancestral plants had stigmas and anthers of the same height as found in many selfing plants, considering that heterostyly arose from self-compatible plants (see introduction), which had lost self-incompatibility probably due to selection favoring selfing. Besides, during simulations, populations experience many different conditions such as dominance of approach or reverse herkogamy before they reach heterostyly.

If Lloyd and Webb (1992a) model (Fig. 8a) is examined with our assumptions, the results should not be very positive. It is because with linear or square-root 
assumptions (Fig. 2) styler dimorphisms (stage at middle of Fig. 8a) is not favored when stigma-anther distances between flowers of the different morphs (connected with arrows in Fig. 8a) is equal to that within the same morph, thus probability of inter- and intramorph pollen deposition is equal. If we find different functions to justify Lloyd and Webb model, we can experimentally examine the different functions and evaluate different hypotheses.

As far as we know this study is first to examine evolution of heterostyly using individual-based model. The model demonstrated that heterostyly could be established from a population with continuous phenotypic variation, which requires more simple assumptions than the previous hypotheses. Only essential genetic effect is linkage between genes for stigma and anther heights. This simple model show clear parallelity between sexual differentiation and speciation, and again supports the view that speciation and sexual dimorphism is different ends of the same evolutional line.

\section{Acknowledgement}

We are grateful to Dr. A. Takenaka, two anonymous reviews and the chief editor for valuable comments on an earlier version of this paper. 


\section{References}

Barrett SCH, ed. (1992) Evolution and function of hterostyly. Springe-Verlag, Berlin.

Barrett SCH (2000) The evolution and function of style polymorphisms in flowering plants. Ann Bot 85:253-265

Barrett SCH (2002a) The evolution of plant sexual diversity. Nat Rev Gen 3:274-284

Barrett SCH (2002b) Sexual interference of the floral kind. Heredity 88:154-159

Barrett SCH (2003) Mating strategies in flowering plants: the outcrossing-selfing paradigm and beyond. Phil Trans R Soc Lond B 358:991-1004

Bawa KS, Beach IH (1983) Self-incompatibility systems in the Rubiaceae of a tropical lowland wet forest. Am J Bot 70:1281-1288

Bertin RI, Sullivan M (1988) Pollen interference and cryptic self-fertility in Campsis radicans. Am J Bot 75: 1140-1147

Bolinick DI, Doebeli M (2003) Sexual dimorphism and adaptive speciation: two sides of the same ecological coin. Evolution 57:2433-2449

Bradshaw HD Jr, Wilbert SM, Otto KG, Schemske DW (1995) Genetic mapping of floral traits associated with reproductive isolation in monkey flowers (Mimulus). Nature 376:762-765

Broyles SB, Wyatt R (1993) The consequences of self-pollination in Asclepias exaltata, a self-incompatible mildewed. Am J Bot 80:41-44

Charlesworth D, Charlesworth B (1979) A model for the evolution of distyly. Am Nat 114:4670-198

Dieckmann U, Doebeli M (1999) On the origin of species by sympatric speciation. Nature 400:354-357 
Fishman L, Kelly AJ, Willis JH (2001) Minor quantitative trait loci underlie floral traits associated with mating system divergence in Mimulus. Evolution $56: 2138-2155$

Ganders FR (1979) The biology of heterostyly. NZ J Bot 17:607-635

Hareder LD, Barrett SCH (1995) Mating cost of large floral displays in hermaphrodite plants. Nature 373:512-515

Harder LD, Wilson WG (1998) A clarification of pollen discounting and its joint effect with inbreeding depression on mating-system evolution. Am Nat 152:684-695

Higashi M, Takimoto G,Yamamura N (1999) Sympatric speciation by sexual selection. Nature 402:523-526.

Hodges SA, Whittall JB, Fulton M, Yang JY (2002) Genetics of floral traits influencing reproductive isolation between Aquilegia formosa and Aquilegia pubescens. Am Nat 159:S51-S60

Juenger T, Purugganan M, Mackey TFC (2000) Quantitative trait loci for floral morphology in Arabidopsis thaliana. Genetics 156: 1379-1392

Kawata M, Toquenaga Y (1994) From artificial individuals to global patterns. Trends Ecol Evol 9: 417-421

Kondrashov AS, Kondrashov FA (1999) Interactions among quantitative traits in the course of sympatric speciation. Nature 400:351-354

Lloyd DG, Webb CJ (1992a) The evolution of heterostyly. In: Barrett SCH (ed) Evolution and function of heterosyly. Springer-Verlag, Berlin, pp 151-178 Lloyd DG, Webb CJ (1992b) The selection of heterostyly. In: Barrett SCH (ed) Evolution and function of heterosyly. Springer-Verlag, Berlin, pp 179-207 Matsumoto YM (2002) Ruby programming Language. Addison Wesley, Boston 
Sakai and Toquenaga 21

Matsumoto Y, Reynolds DL (2001) Ruby in a Nutshell. O’Reilly, Beijing

Orr HA (2001) The genetics of species differences. Trends Ecol Evol 16:343-350

Richards AJ (1997) Plant breeding systems, 2nd edn. Chapman \& Hall, London

Seavey SF, Carter SK (1993) Self-fertility in Epilobium obcordatum (Onagraceae). Am J Bot 81:331-338

Waser NM, Price MV (1991) Reproductive costs of self-pollination in Ipomopsis aggregata (Polemoniaceaea). Am J Bot 78:1036-1043 


\section{Figure legends}

Fig. 1. The heterostylous genetic polymorphism (distyly). Compatible pollinations are indicated by the arrows. The dimorphism is controlled by a single locus with two alleles. Usually the pin morph (right) with higher stigma is of genotype ss and the thrum morph (left) with $\underline{\text { Ss }}$.

Fig. 2. Diagrams showing relationships between reproductive success and phenotypic traits of individual and mating pair under linear (a) and square-root (b) assumptions. (1) Seed production or probability of pollen removal monotonously increase with stigma-anther separation within a flower. (2) Probability of successful pollen deposition monotonously decreases with the difference between anther height of the pollen donor and stigma height of the recipient. Combined effects of (1) and (2) predict relative reproductive success of individual through pollen or seeds depending on expected stigma-anther separation when combination between anther and stigma within a flower is random.. Optimal height difference, which provides the highest reproductive success is indicated by arrows.

Fig. 3. Schema illustrating relationships between genetic values and phenotypic values. The stigma height $\left(H_{s}\right)$ is determined by the genetic values for stigma height $\left(s_{1}\right.$ and $s_{2}, s_{2}>s_{1}$ in this case) and genetic values for dominance effect on the stigma height ( $d_{s 1}$ and $d_{s 2}$ ). (a) When there is no dominance effect (Conditions 1-4 in Table 1), $H_{s}$ is at the middle (average) of $s_{1}$ and $s_{2}$ (Conditions 5,6). (b) When the dominance effect is present, $H_{s}$ is somewhere between $s_{1}$ and $s_{2}$ depending on the average of $d_{s 1}$ and $d_{s 2}$. 
When the average is larger than 0.5 , the phenotypic value is closer to $s_{2}$, while when it is smaller the value is closer to $s$.

Fig. 4. Schema showing our definition of heterostylous populaion. When the location of the two cells with the most and second most individuals, $\left(H_{s 1}, H_{a 1}\right)$ and $\left(H_{s 2}\right.$, $H_{a 2}$ ), are located the different sides of the line $H_{a}=H_{s}$ (i.e. stigma is higher in a cell and it is opposite in the other), and correlation of stigma and anther heights $\left(\operatorname{Cov}\left[H_{s}, H_{a}\right]\right)$ of the two cells are negative (i.e. size relationships in stigma height between the two cells are opposite in anther height), the population is judged to be heterostylous.

Fig. 5. Frequency distribution of combination of stigma and anther heights at the end of 200 simulation runs under the linear (a) and square-root assumptions (b) (Conditions 1 and 2 in Table 1). The brightest color responds to the highest frequency. The white dotted lines indicate optimal combinations of stigma and anther heights in monomorphic populations under the two conditions.

Fig. 6. An example of establishment of heterostyly under the condition 4 (Table 1). The upper two graphs show temporal changes in distribution of stigma and anther heights in a run. The brightest color responds to the highest frequency. Linkage between stigma and anther genes is selected for and getting stronger during the establishment of dimorphy, as shown in the graph second from the bottom. After 400 generations, the populations is dominated by two morphs, which have stigma and anthers reciprocally arranged. In this case homozygotes have higher stigmas and lower anthers (pin) and heterozygotes have lower stigmas and higher anthers (thrum).

Fig. 7. An example of establishment of heterostyly under the condition 5 (Table 1). The 
brightest color responds to the highest frequency. The upper two graphs show temporal changes in distribution of stigma and anther heights in a run. Strong linkage between stigma and anther genes, and stronger dominance (for higher value for stigma, for lower value for anther in this run) are selected for during the establishment of heterostyly, as shown in the graph second from the bottom. After 400 generations, the populations is dominated by two morphs, which have stigma and anthers reciprocally arranged. In this case heterozygote has higher stigmas and lower anthers (pin) and homozygote has lower stigmas and higher anthers (thrum).

Fig. 8. The postulated scenarios for the evolution of heterostyly. (a) Lloyd and Webb (1992a) assume approach herkogamy as ancestral condition (left), subsequent invasion and spread of a mutant with higher anthers (middle), and establishment of reciprocal herkogamy (right). On the other hand, (b) this study assumes populations with stigmas and anthers with some variations as ancestral (right), which evolve into dimorphism in stigma and anther heights and heterostylous population (left). 


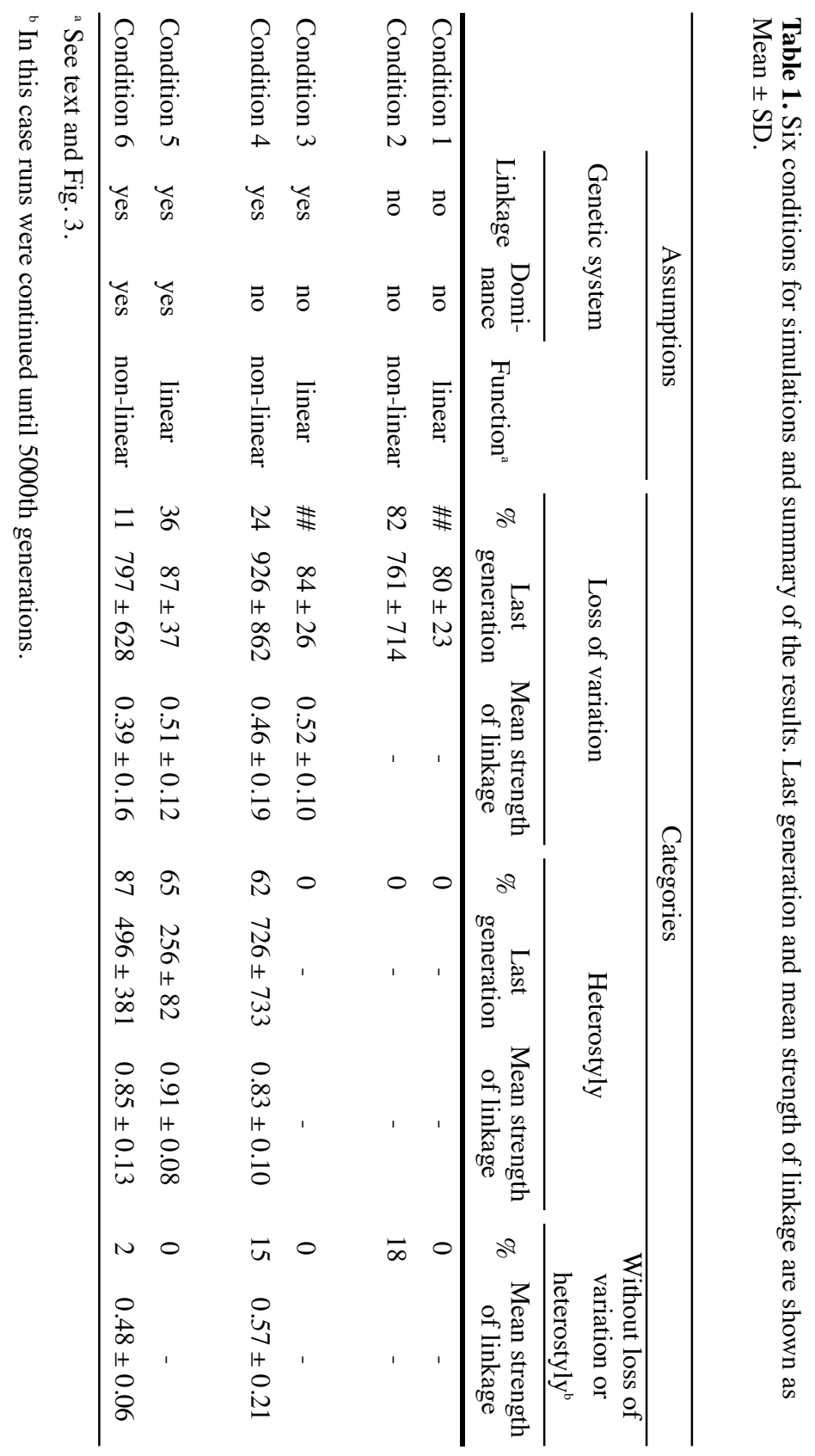




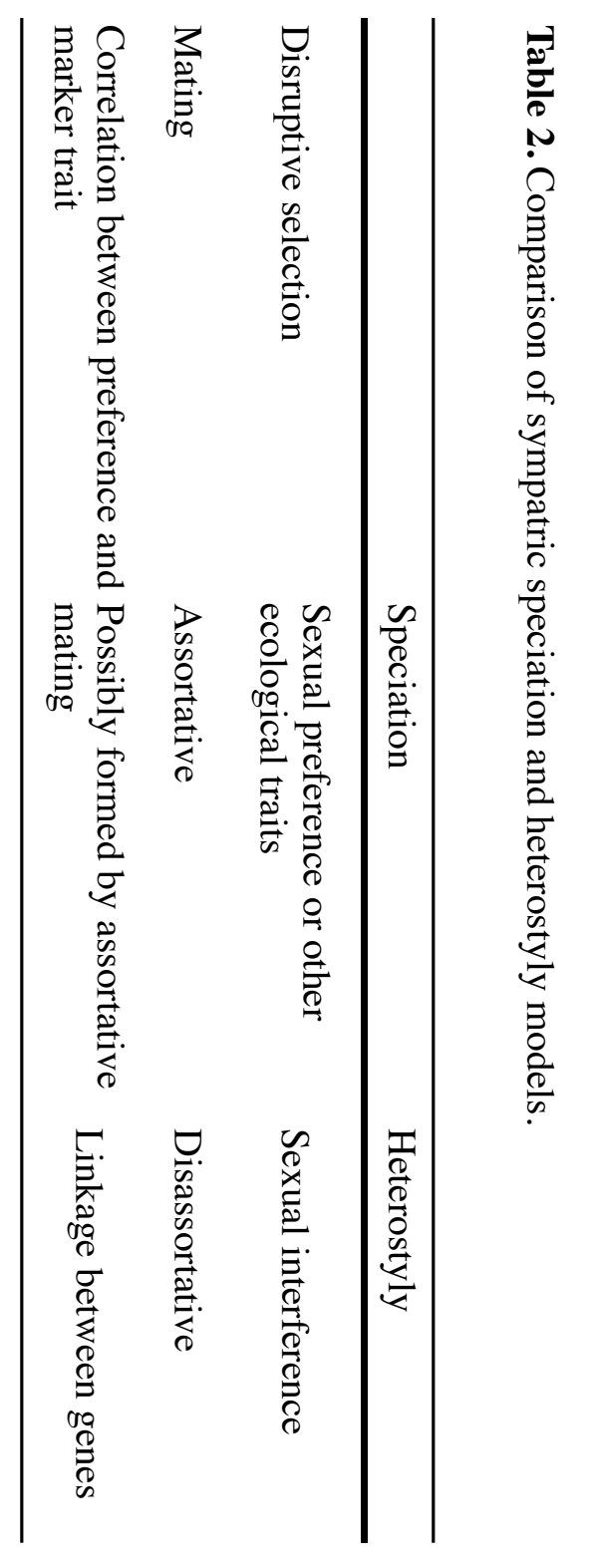




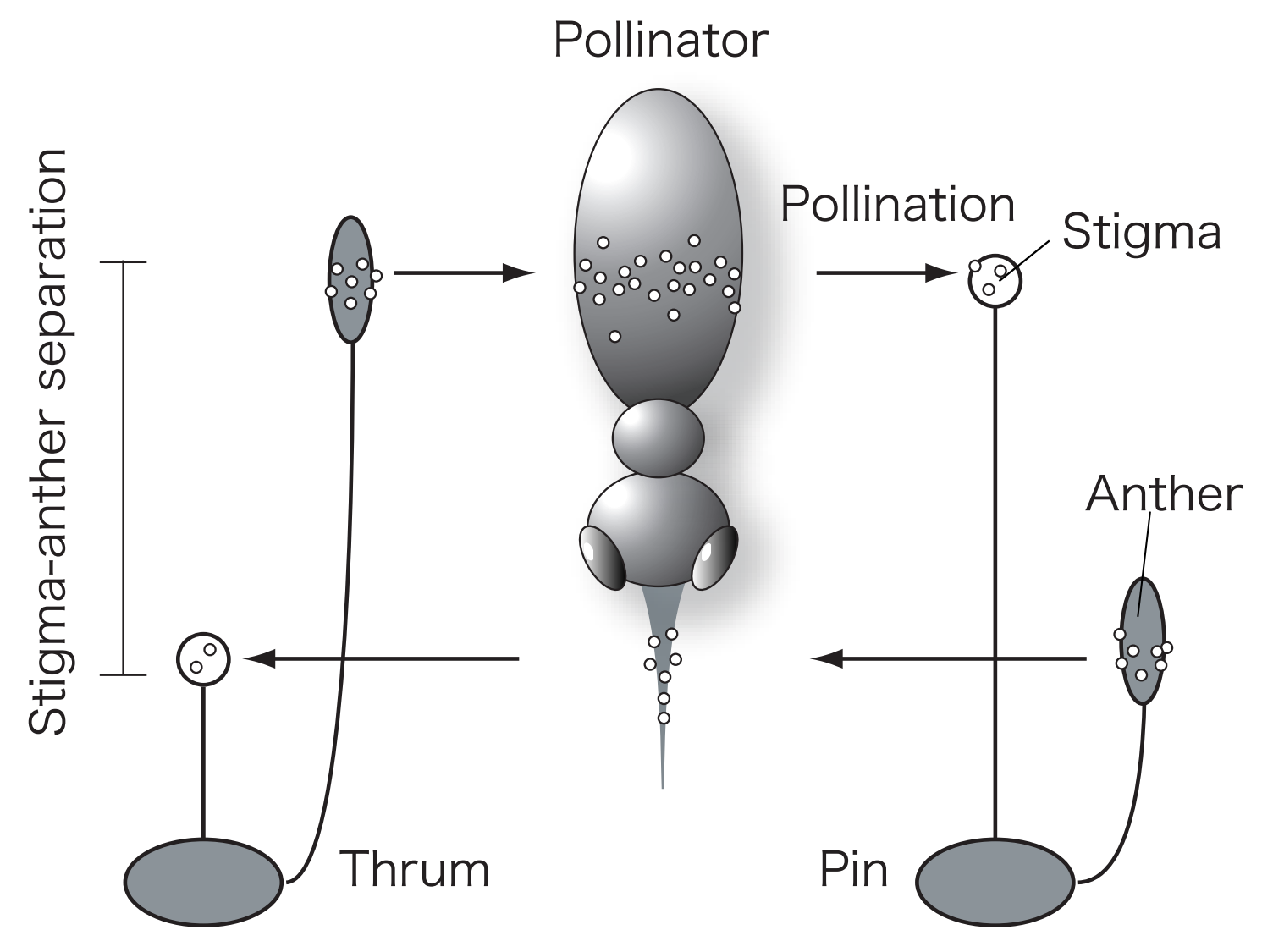




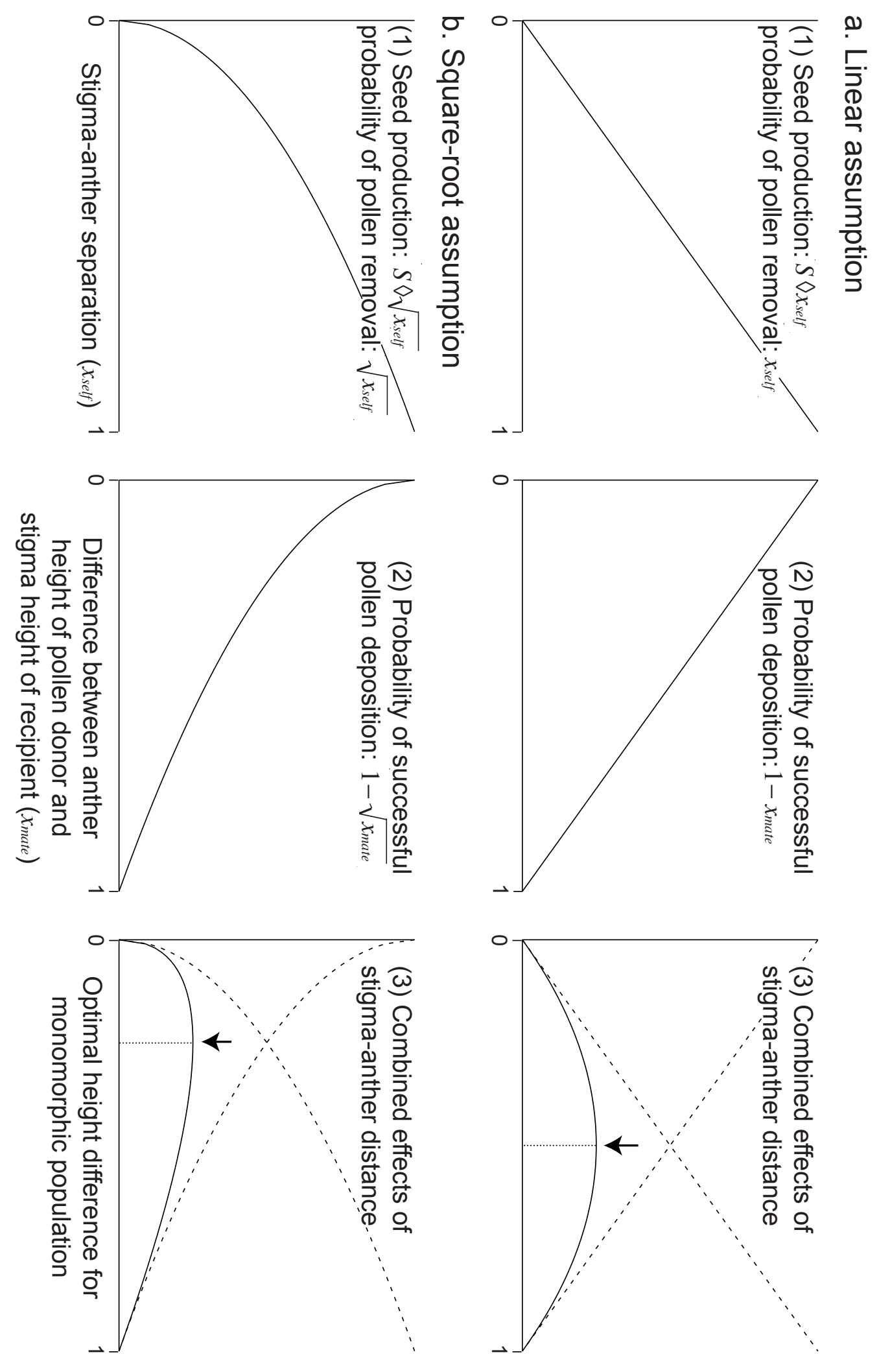




\section{a. $H_{\mathrm{s}}$ without dominance effect}

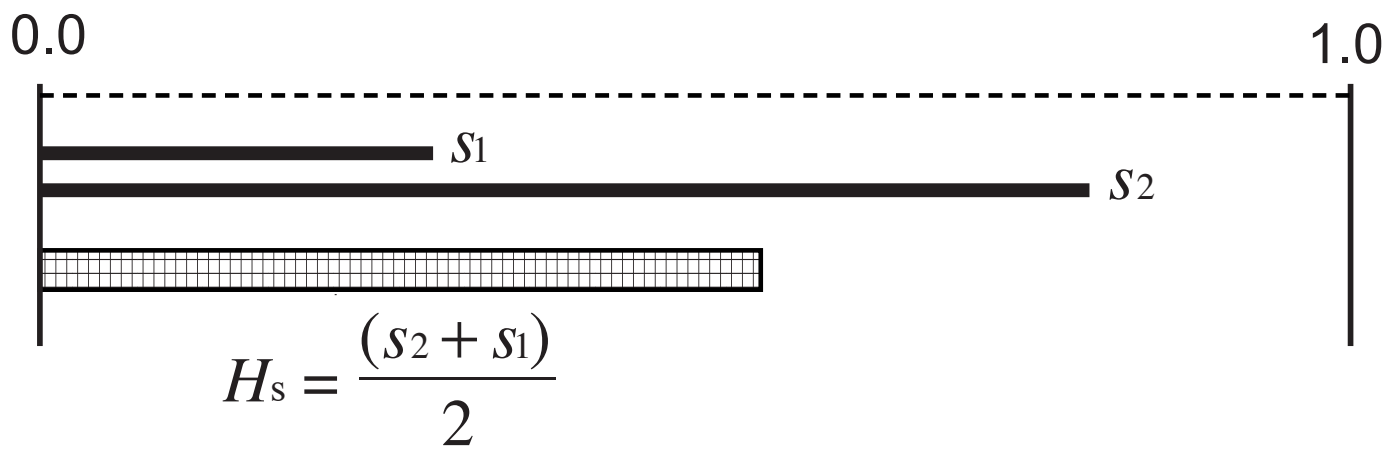

\section{b. $H_{\mathrm{s}}$ with dominance effect $\left(s_{2}>s_{1}\right)$}

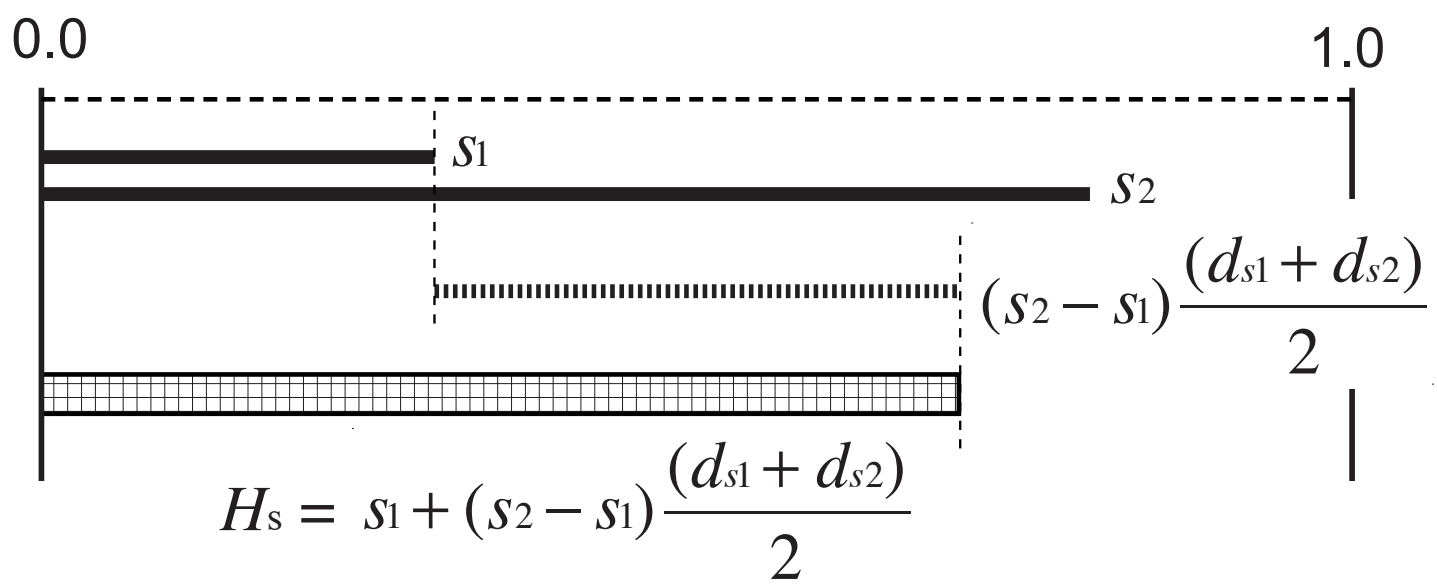




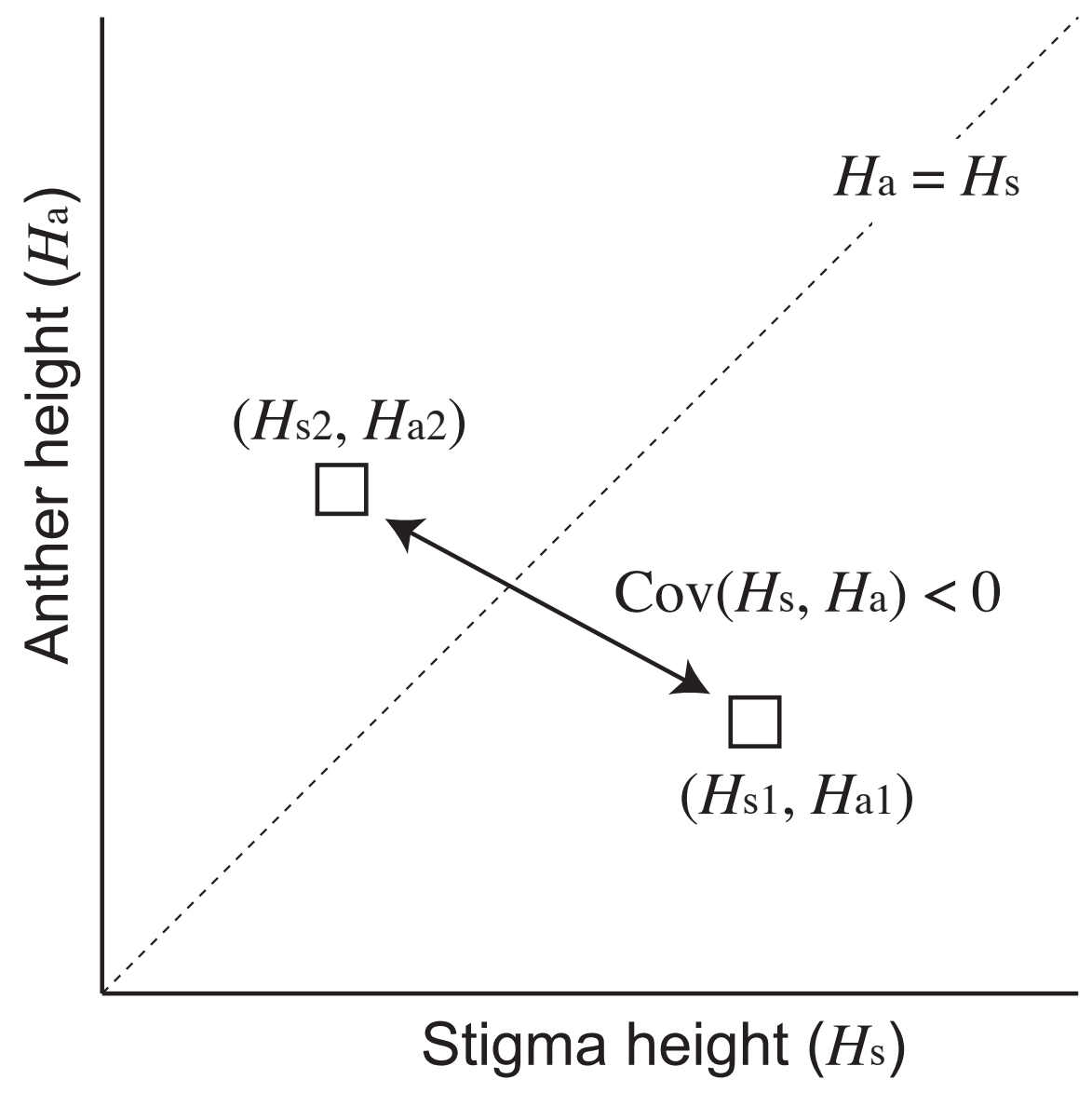




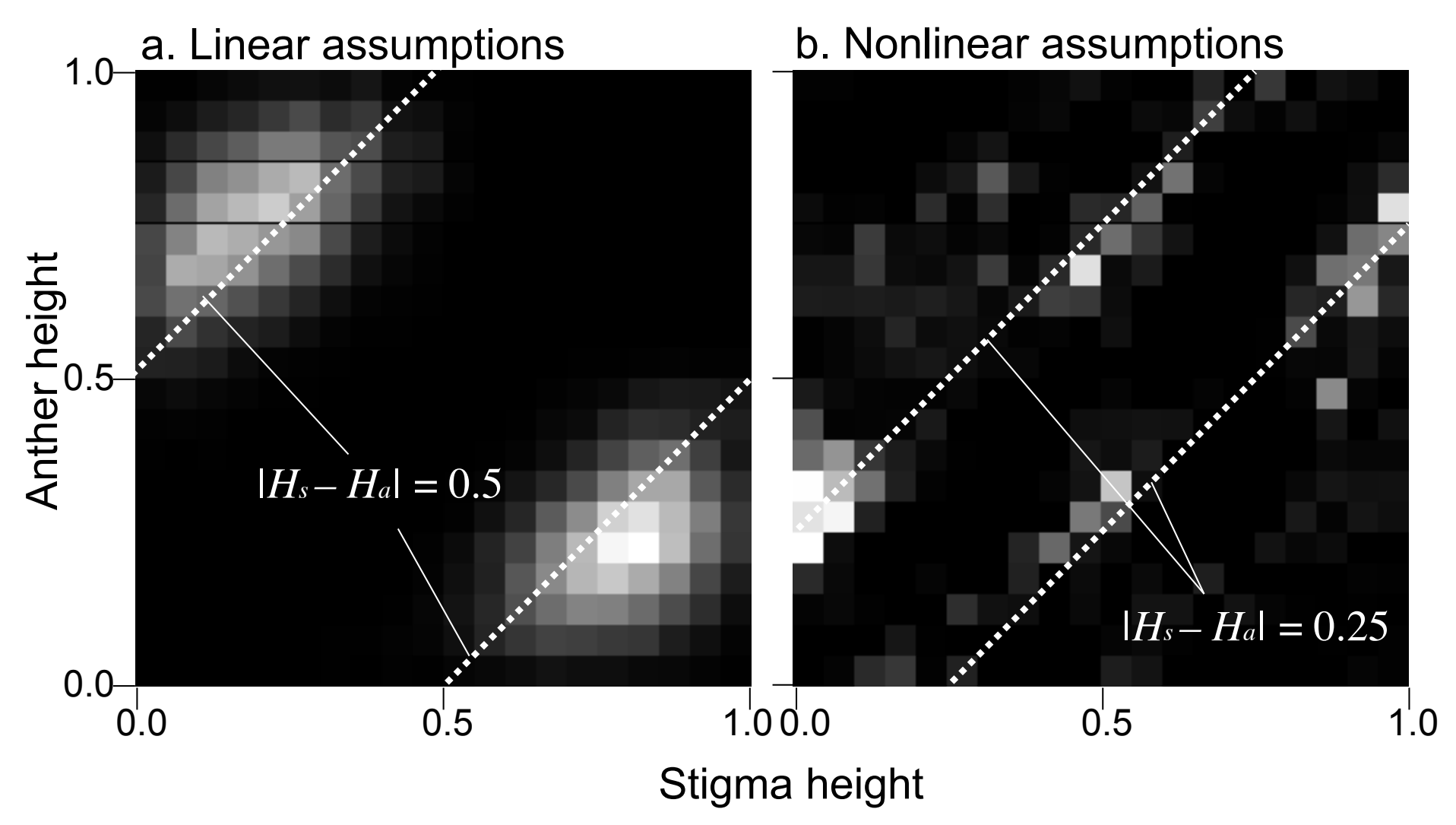



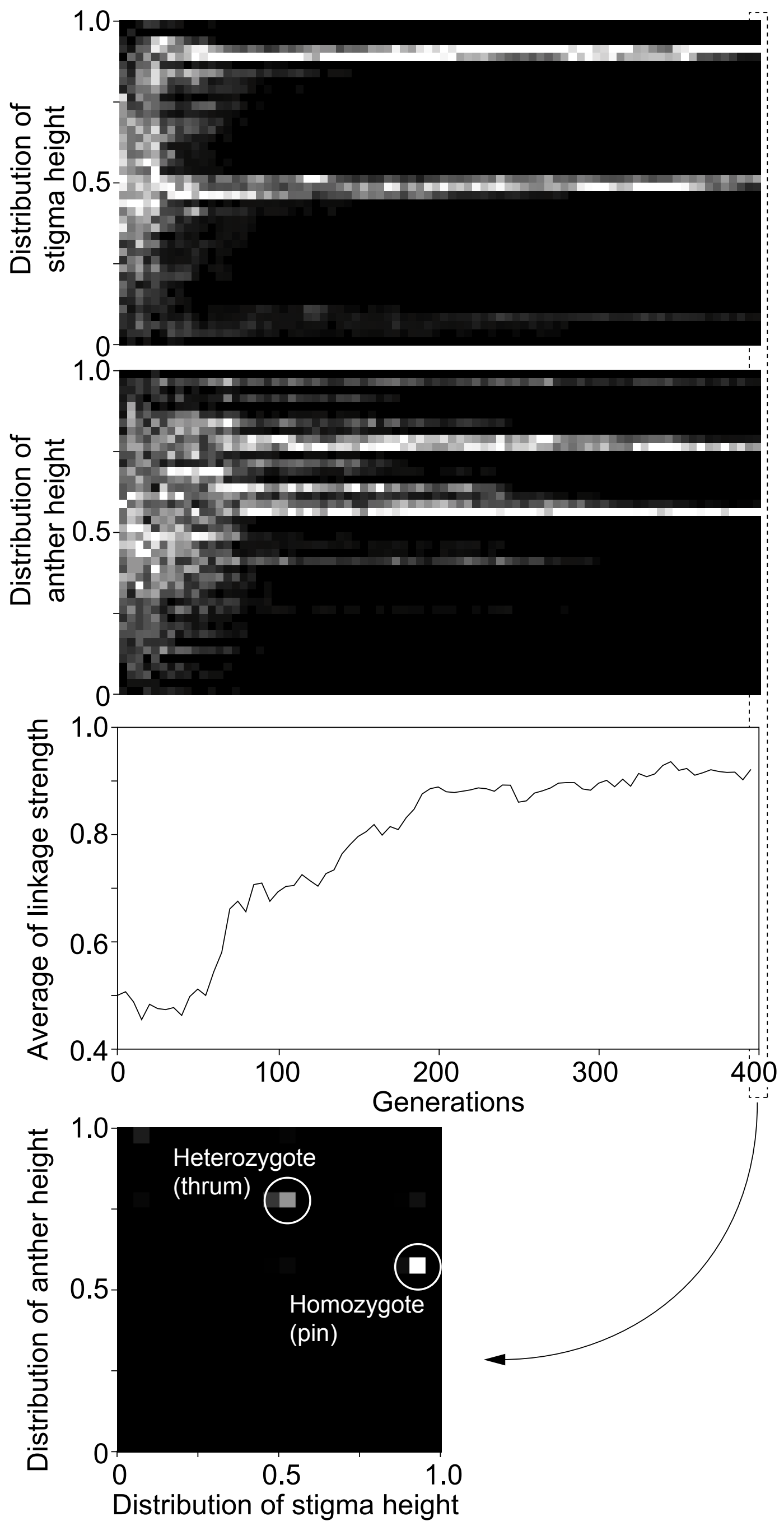

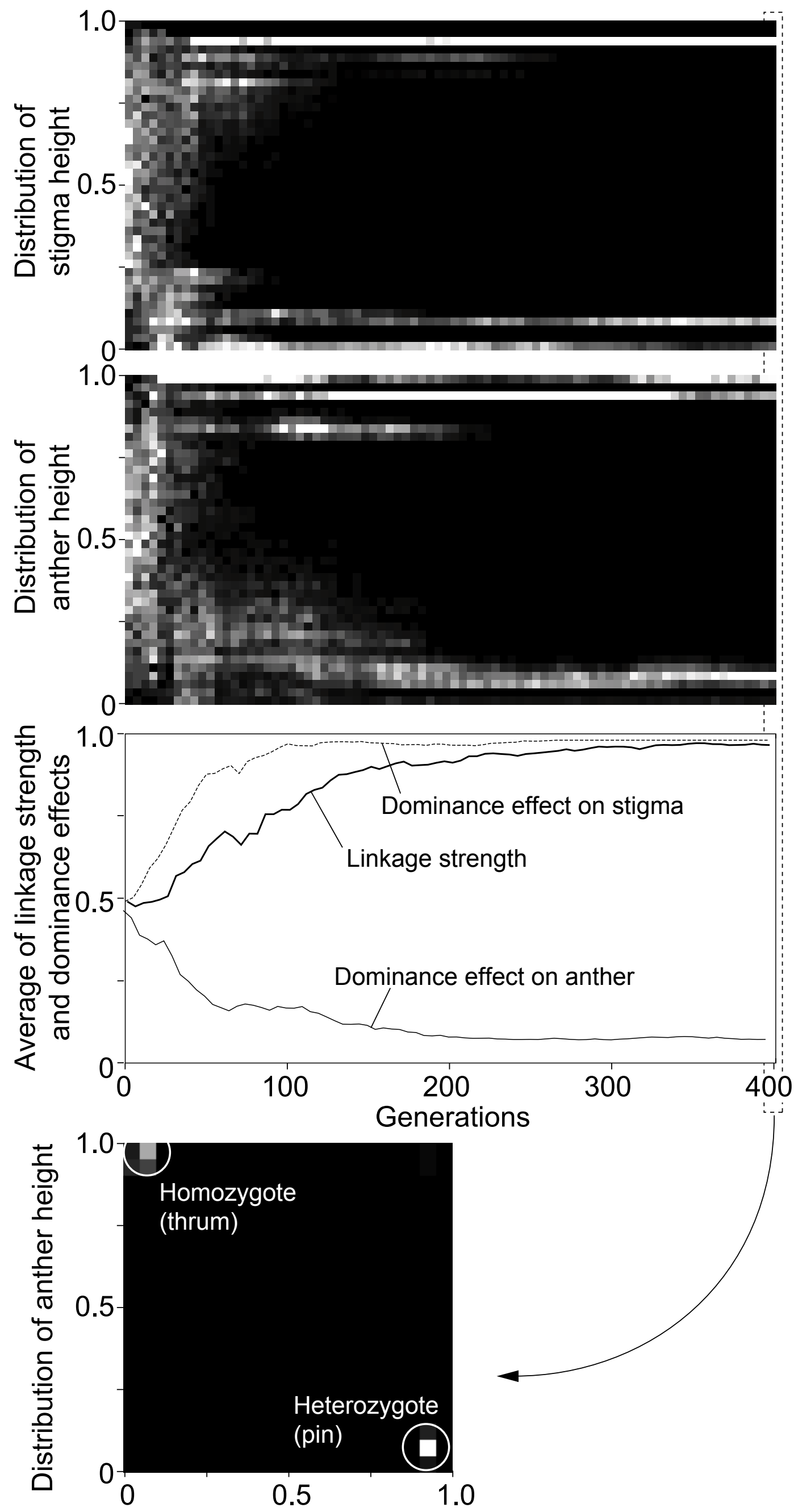

Distribution of stigma height 
a. Lloyd and Webb model

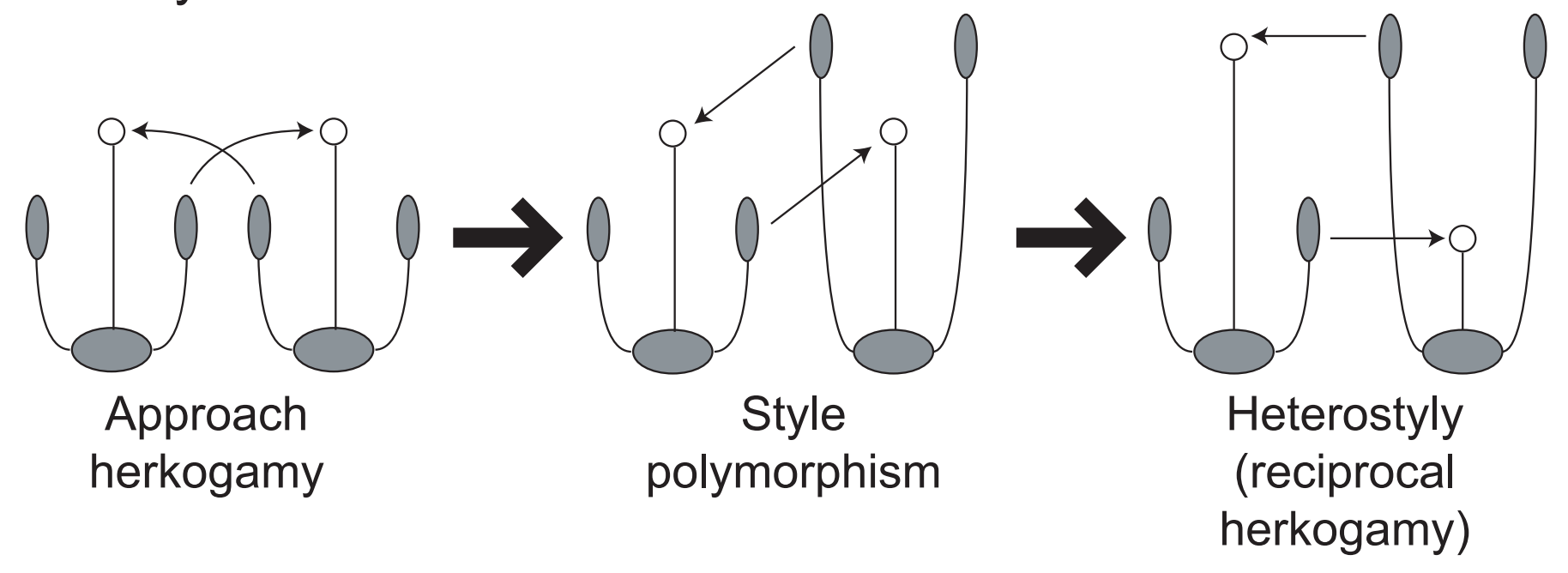

b. This study

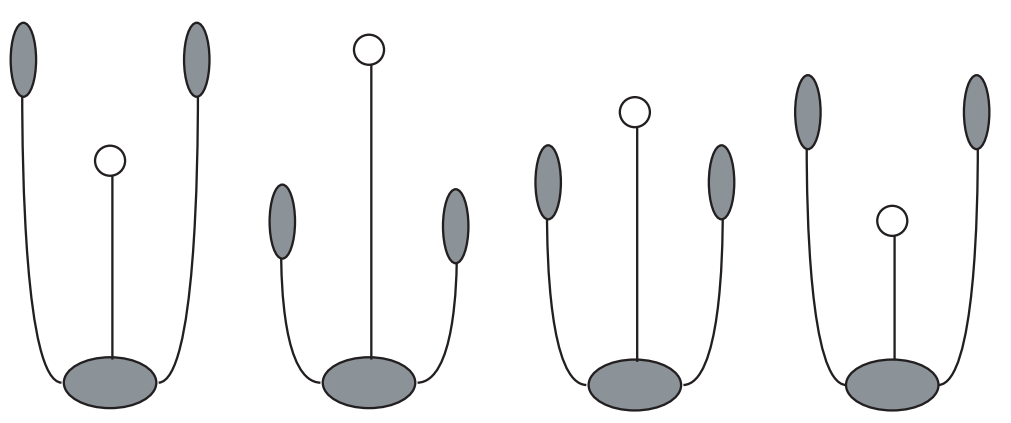

Stimgma and anther heights with variation

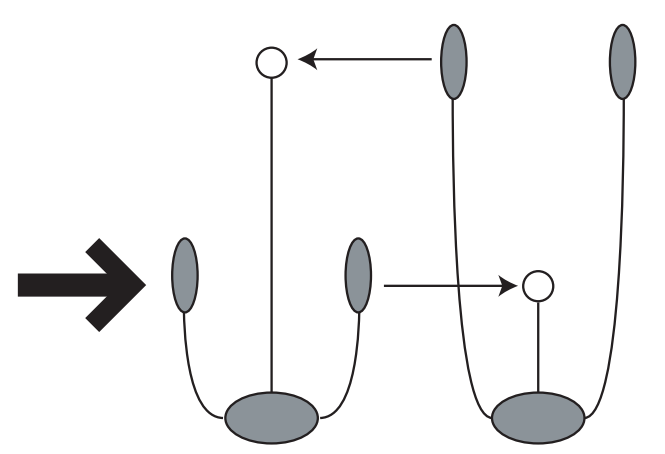

Heterostyly (reciprocal herkogamy) 